\title{
AODVH: Ad hoc On-demand Distance Vector Routing for Hybrid Nodes
}

\author{
Yasmin Jahir $^{1}$, Mohammed Atiquzzaman ${ }^{2}$, Hazem Refai ${ }^{1}$, Peter G. LoPresti ${ }^{3}$ \\ ${ }^{1}$ School of Electrical and Computer Engineering, University of Oklahoma, Norman, OK. \\ ${ }^{2}$ School of Computer Science, University of Oklahoma, Norman, OK. \\ ${ }^{3}$ Department of Electrical Engineering, The University of Tulsa, Tulsa, OK. \\ Email: $\{$ yasmin, atiq\}@ou.edu
}

\begin{abstract}
Ad hoc networks are characterized by nodes which are usually connected by low data rate Radio Frequency (RF) links when compared to optical links. RF links make it challenging for transmitting video and running high bandwidth applications over ad hoc networks. The objective of this paper is to develop a novel routing protocol for Ad hoc networks consisting of hybrid nodes using high bandwidth Free Space Optical (FSO) and RF links, with FSO being the primary link and RF as backup in case of FSO link failures. Our protocol, called Ad hoc Ondemand Distance Vector hybrid (AODVH), computes multiple "FSO only" paths to ensure high data rate communication, and uses "hybrid paths" consisting of FSO and RF links as backups. AODVH can be used to design a rapidly deployable, reliable and high bandwidth communication infrastructure for re-establishing communication following a disaster. We evaluate the performance of AODVH using ns-2 simulations and compare with three other Ad hoc routing protocols. Results show that AODVH performs better in terms of packet loss, end-to-end delay, overhead, packet delivery ratio, route discovery frequency and throughput when compared to the other three protocols.
\end{abstract}

\section{INTRODUCTION}

A rapidly deployable, reliable and high bandwidth network is needed during natural or man made disasters resulting in breakdown of telecommunications infrastructure [1]. We propose such a network architecture, called Disaster Area Wireless Network (DAWN), consisting of helium-filled balloons carrying routers. The balloons form an ad hoc network up in the sky using Free Space Optical (FSO) and Radio Frequency (RF) links.

An ad hoc network is characterized by a multihop, temporary network consisting of a set of nodes that do not rely on any preexisting infrastructure support [2] and usually communicate with each other using RF links. RF links have low bandwidth and, therefore, are challenging for high bandwidth applications such as multimedia transmission.

A Free Space Optical (FSO) link has significantly higher bandwidth and lower error rate [1] over RF links. However, link unavailability remains a big challenge for FSO links due to environmental phenomenon such as absorption, scattering (fog) and shimmer [3]. Because of this intermittent characteristic of FSO links, it is not always possible to maintain " $F S O$ " links. Hybrid nodes consisting of FSO and RF links, with RF

This work was supported by National Science Foundation grant no. 0725801 . links serving as backup in case of FSO link unavailability, can be used to overcome the above limitation of FSO links. The challenge, however, is to design multipath routing protocols which will take into account the varying characteristics of FSO and RF links. Multiple paths increase fault tolerance and reduce control message overhead [4], thus improving the effective bandwidth among communicating pairs.

Our objective is to develop a multipath on-demand distance vector routing protocol for hybrid nodes, called multipath Ad hoc On-demand Distance Vector Hybrid (AODVH). AODVH differs from other Ad Hoc routing protocols (DSR [5], OLSR [6], AODV [7], AOMDV [8], AODVM [9], etc.) mainly in two aspects:

- Current single path Ad Hoc routing protocols require considerable time to find new routes in case of link failures, and are thus unsuitable for DAWN where link failure is frequent with hybrid nodes having intermittent FSO links;

- Current multipath protocols do not support routing over hybrid links (FSO and RF) with different link characteristics.

Existing multipath Ad hoc routing algorithms [2], [8], [9] are extensions of unipath routing protocols viz. DSR [5] and AODV [7]. Lee et al. [2] developed a split multipath routing protocol with maximally disjoint paths for homogeneous RF nodes. Marina et al. [8] developed a loop free and link-disjoint multipath routing protocol (AOMDV). Zhenqiang et al. [9] proposed AODVM to discover multiple node-disjoint paths to achieve reliability in path setup for homogeneous RF nodes. The above [2], [8], [9] are based on homogeneous nodes and hence are not suitable for our hybrid nodes having heterogeneous link characteristics. Our proposed protocol (AODVH), differs from other multipath routing protocols because it considers hybrid nodes with heterogeneous link characteristics. Bilgi et al. [10] and Yuksel et al. [11] proposed nodes with FSO links only, and hence differ from our requirements of hybrid nodes.

The main contribution of this paper is to develop a novel multipath Ad Hoc routing protocol for computing multiple "FSO only" paths to ensure faster communication among hybrid nodes using FSO and RF links in DAWN.

We have simulated AODVH in ns-2 and compared the results with unipath AODV [7] and two multipath protocols 
(AOMDV [8] and AODVM [9]).

The rest of the paper is organized as follows. We review AODV in Sec. II-A and AOMDV and AODVM in Secs. II-B and II-C, respectively. In Sec. III, we describe our proposed AODVH protocol. Our ns-2 simulation setup is given in Sec. IV, followed by the performance evaluation of AODVH and its comparison with AODV, AODVM, and AOMDV in Sec. V. Finally, concluding remarks are presented in Sec. VI.

\section{Ad hoc On-Demand Distance Vector Routing}

In this section, we briefly discuss AODV, one of the wellstudied unipath reactive routing protocol, and two multipath extensions of AODV viz. AOMDV and AODVM.

\section{A. $A O D V$}

AODV combines the use of DSDV [12] with an on-demand route discovery technique as described below:

Route Discovery: The source initiates a route discovery process by flooding a RREQ message whenever a route to the destination is needed. Upon receiving a RREQ, a node checks to see if it is the destination or whether it has a fresh enough route to the destination. If either case is true, the node generates a route reply (RREP) message, which is sent back to the source along the reverse path. Each node along the reverse path sets up a forward path to the node it received the RREP from.

Route Maintenance: When a node detects a broken link while forwarding a packet to the next hop, it generates a route error (RERR) message. The RERR erases all routes that use the link along the way to the source and the source initiates a new route discovery process. Also stale routes are deleted from the routing table if they are unused for a certain period of time.

\section{B. $A O M D V$}

AOMDV is the multipath extension for AODV that computes multiple link disjoint paths between nodes.

Route Discovery: AOMDV's route discovery procedure finds link disjoint paths where no link can be common among the multipaths.

Route Maintenance: The fault tolerance of AOMDV sometimes is lower than AODVM because in high mobility or stressful situations, the chances of losing multiple paths are higher as a node may contain several links to form multiple paths.

\section{C. $A O D V M$}

AODVM is a multipath extension to AODV to allow discovery of multiple node-disjoint paths where no node can be common.

Route Discovery: When the intermediate nodes receive multiple RREQ messages from previous nodes, instead of discarding the duplicate RREQ messages, they record the information in the RREQ table and, unlike AODV or AOMDV, refrain from sending a RREP back to the originating source to make sure all the RREQs reach the destination.
Route Maintenance: Intermediate node forwards a RREP through the neighbor in the RREQ table from which the path to the source is the shortest. The corresponding entry of that neighbor is deleted from the RREQ table. When other nodes overhear any node forwarding a RREP, they delete the entry corresponding to that node from their RREQ tables.

\section{AODVH - PROPOSED HYBRID AODV}

Based on AODV (Sec II-A) and AODVM (Sec II-C), we describe our proposed multipath on-demand routing protocol, AODVH, for hybrid nodes.

\section{A. Double Interface}

We implement a hybrid node structure that supports two interfaces having RF and FSO characteristics. We opted for using multiple instances of the wireless channel at the node level and used the MAC features of NS-2 to get the address of the interface the message arrives on. For broadcast messages, we transmit messages through both the interfaces but for unicast transmission, we direct messages through the appropriate interface.

\section{B. Route Request Message (RREQ)}

The source sends RREQ message to both FSO (if available) and RF links. Like AODVM, intermediate nodes do not discard duplicate RREQ messages. Route Request (RREQ) tables are constructed that store the necessary information about source of the RREQ, destination, neighbor list, hops to source, expiration timer, FSO and RF flags. The nodes set the flags for FSO and RF in their respective RREQ tables based on the interface the message has been received. For example, in Fig. 1, during the first stage of RREQ message, nodes 1, 2 and 3 receive RREQ from the source.

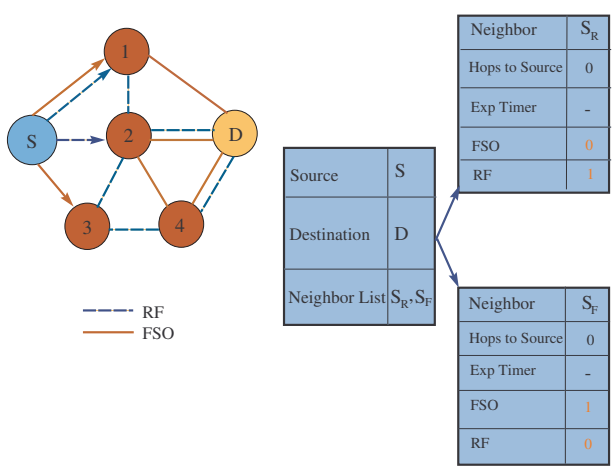

Fig. 1. Route Request (RREQ) table for node 1 (Stage 1).

Nodes 2 and 3 also set up their respective RREQ tables the same way. During stage 2 of RREQ message, these intermediate nodes send the RREQ messages received from the source to their neighbors. Like before, the neighbors update their respective RREQ tables. In this way, the duplicate RREQ messages, containing information regarding the type of interface (FSO, RF or both) used along the path, reach the destination node. 


\section{Route Reply Message (RREP)}

Upon receiving RREQs from its neighbors, the destination node starts generating RREPs. These RREPs route through the reverse paths that were created during RREQ stages. A forward path is setup during the generation of the RREP message from source to the destination for data delivery. Due to the mutlipath nature of AODVH, the destination node replies to all RREQs it receives from the intermediate nodes and tries to find "FSO only" paths first followed by "Hybrid paths" if "FSO only path" is not available. After forwarding the first RREP from node 1, the entry for node 1 is deleted from the RREQ tables of other intermediate nodes to achieve node disjointness. When all the RREPs reach the source $\mathrm{S}$, we have multiple paths to send data from source to destination $\mathrm{D}$, with the first path being the primary one. The scenario is shown in Fig. 2.

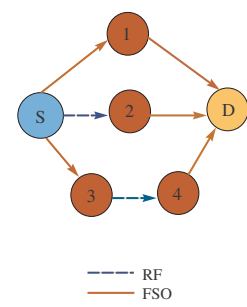

Fig. 2. Forward Path set up from source S.

\section{D. "FSO Only" Path}

For our proposed network architecture, we prefer "FSO only" paths for high bandwidth and adapted an FSO enhancement [10] for one of the interfaces in our hybrid node structure that uses channel information to extract the meta data information present in the packet headers to determine the divergence angle, specific position and normal of the sending transceiver. In this process, the wireless channel goes through every node in the network, to find out if the sender can see the candidate (destination or intermediate) node. If the two given nodes can see each other, a message is scheduled for reception by the transceivers in the candidate node for delivery to the upper layers.

Auto-alignment circuitry has been used with multiple transceivers for FSO transmitters pointing at the targeted destination. When the destination node starts receiving the RREQs, it checks the FSO and RF flags in the RREQ messages, and tries to generates RREPs to FSO medium. We use hello messages to determine local connectivity. Failure to receive any hello message from a neighbor for several time intervals indicates that the neighbor is no longer within transmission range, and Route Error (RERR) is generated. FSO medium access is faster than RF, so the hello packet interval is reduced and in case of a FSO path failure, the alternative path is picked up almost immediately.

\section{Simulation Setup}

We simulated our proposed protocol (AODVH) using Network Simulator (ns-2) [13]. Our objective is to evaluate the effectiveness of AODVH relative to AODV, AODVM and AOMDV, especially when route failures occur due to mobility. We also evaluate the effectiveness of "FSO only" paths and "Hybrid" paths of AODVH over "RF only" paths of AOMDV and AODVM in terms of throughput.

The Monarch research group in Carnegie Mellon University (CMU) added extra features in ns-2 to support multihop wireless networks [8]. We used the FSO characteristics model developed at University of Nevada (Reno) [10] for one interface. Hybrid nodes were implemented with double interfaces consisting of FSO and RF links. Values of relevant simulation parameters for hybrid nodes with two interfaces are summarized in Table I.

TABLE I

SIMULATION PARAMETERS

\begin{tabular}{ll}
\hline Network Size & $1000 \mathrm{~m} \mathrm{x} 1000 \mathrm{~m}$ \\
Number of Nodes & 16 \\
Simulation Time & $100 \mathrm{sec}$ \\
Propagation Type1 & Free Space Optical \\
Propagation Type2 & Two Ray Ground \\
Channel capacity & $2 \mathrm{Mbps}$ \\
Node Transmission Range & $250 \mathrm{~m}$ \\
\hline
\end{tabular}

We averaged the outcomes of five runs for the same node speed and generated the plots. Traffic pattern consists of FTP/TCP connection between a source and destination pair. The data packets have a fixed size of 1000 bytes in all the experiments. The maximum number of multipath routes was set to three [8]. The random way point mobility model was used to simulate node movements. We generated different random mobility scenarios using different node speeds. The nodes in this scenario move along a zigzag path that consists of straight lines between points where each point is chosen by uniform distribution [14].

\section{REsUlts}

In this section, we evaluate the effectiveness of AODVH and compare with those of AODV, AODVM and AOMDV using packet loss, end-to-end delay, route discovery frequency, routing overhead and throughput as the performance metrics.

Packet Loss: We measure packet loss by the percentage of data packets that are dropped. Fig. 3 shows the packet loss of AODVH, AODV, AODVM and AOMDV as a function of node speed. A higher speed results in larger number of link breaks. In AODVH, AOMDV and AODVM, the source will have alternative routes to the destination; hence packet losses occur mostly at the intermediate nodes for these protocols. For AODV, routes will fail more frequently with increased node mobility resulting in larger number of route discoveries from the source to find a route and higher packet loss.

For low mobility, AODVH performs significantly better than other protocols (50\% less packet loss than AODV, and 44\% less than AOMDV and AODVM). This is because of the Free Space Optical propagation model for "FSO only" path where high transmit power and high frequency of optical links result in lower packet loss than RF used in other protocols.

End-to-End Delay: End-to-end delay is the sum of all possible delays encountered by a packet between a source 


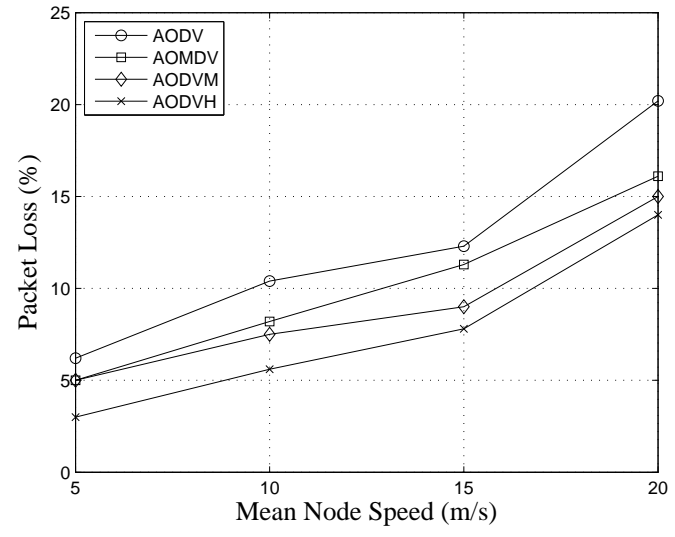

Fig. 3. Effect of mobility on packet loss.

and destination. Fig. 4 compares average end-to-end delay of AODVH with the other three routing protocols.

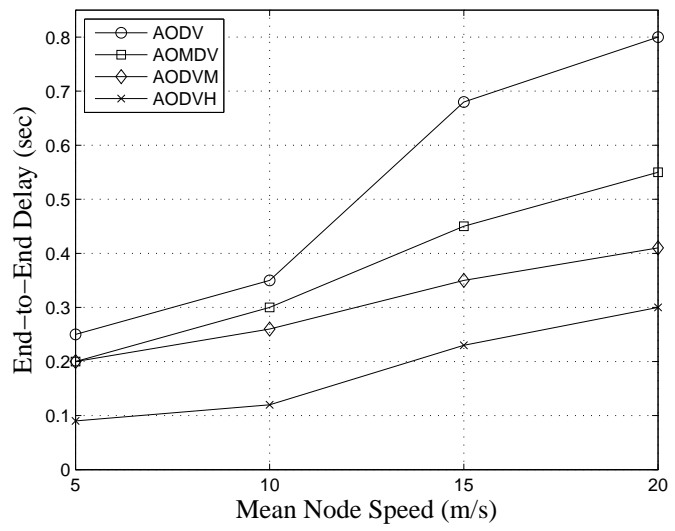

Fig. 4. Effect of mobility on end-to-end delay.

At a speed of $10 \mathrm{~m} / \mathrm{s}$, AODVH achieves $65 \%, 60 \%$ and $55 \%$ reduction in end-to-end delay when compared to AODV, AOMDV and AODVM, respectively. The regular maintenance of "FSO only" paths in AODVH results in increased availability of valid alternate paths when primary path breaks. Also node-disjoint multipath AODVH and AODVM performs better than link-disjoint AOMDV due to the fact that node failure in a link-disjoint path can affect several paths that are common to the failed node.

Route Discovery Frequency: We define route discovery frequency by the number of route requests initiated by the source per second. Fig. 5 shows the route discovery frequency of the four protocols as a function of node mobility.

AODV has the highest number of route requests whereas the multipath feature of AOMDV, AODVM and AODVH results in fewer route discoveries than AODV. AODVH performs the best due to availability of FSO paths that offer higher fault tolerance, resulting in lower route discovery frequency even for higher mobility.

Packet Delivery Ratio: We measure packet delivery ratio by the ratio of data packets delivered at the destination to data

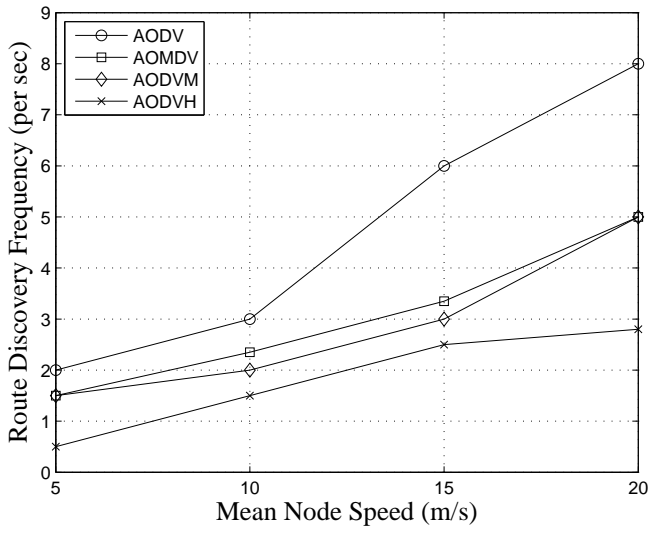

Fig. 5. Effect of mobility on route discovery.

packets transmitted by the source. Fig. 6 shows the packet delivery ratio of AODVH, AODV, AOMDV and AODVM. At lower speeds, for example $5 \mathrm{~m} / \mathrm{s}$, all the protocols perform well. At a higher speed (say, $20 \mathrm{~m} / \mathrm{s}$ ), the performance of AODVH is reduced to $86 \%$ because of higher losses resulting of larger number of link failures; AODVH is still better than AODV, AOMDV and AODVM by 15\%, 10\% and 8\%, respectively.

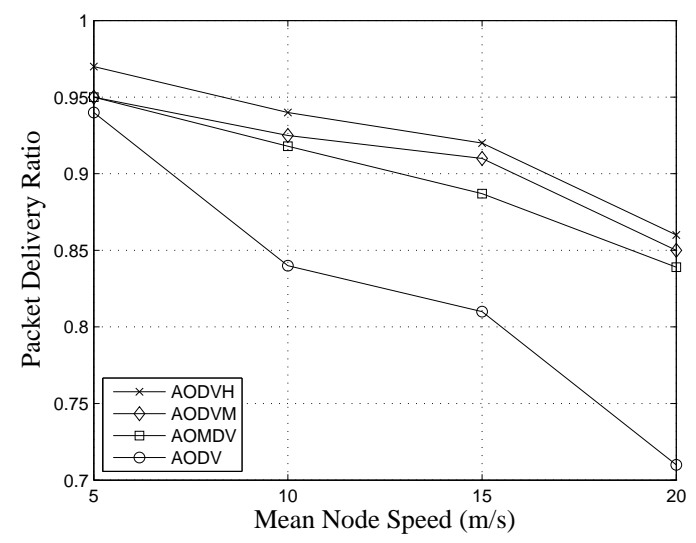

Fig. 6. Effect of mobility on packet delivery.

Routing Overhead: Routing overhead is measured by the total number of per hop transmission of routing messages per second. Fig. 7 compares routing overhead of AODVH with other protocols as a function of node mobility. AODVH, AOMDV and AODVM have lower overhead than AODV due to less route discoveries required in multipath option. Although AODVH performs better than other protocols, the overhead is still not small due to the additional RREP messages at the intermediate nodes to create "FSO only" paths.

Throughput: We measure throughput by the total number of bits received at the destination per unit time. Fig. 8 compares the throughput of AODVH ("FSO Only" paths) with AODVM (RF only paths) and AODV (RF only paths). The throughput for "FSO only" paths of AODVH is much higher (75\% more) than throughput of AODV and AODVM because of the Free 


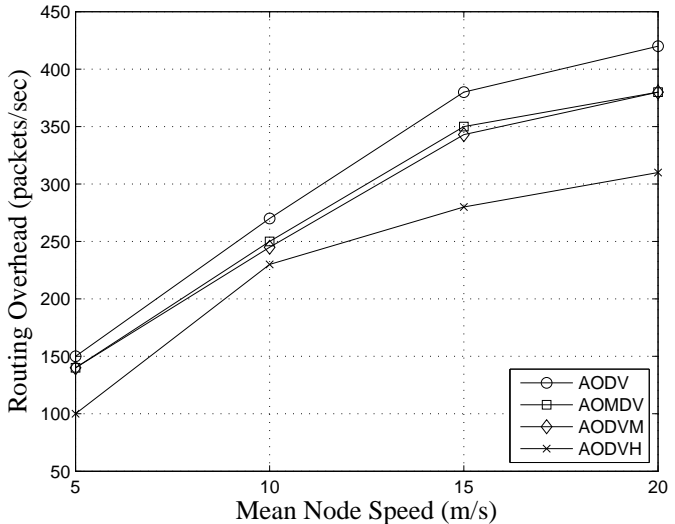

Fig. 7. Effect of mobility on routing overhead.

Space Optical propagation model for "FSO only" path where high transmit power and high frequency of optical links result in lower packet loss than RF used in other protocols. AODVM and AOMDV perform better than AODV due to availability of alternate path in case of link failure of the primary path.

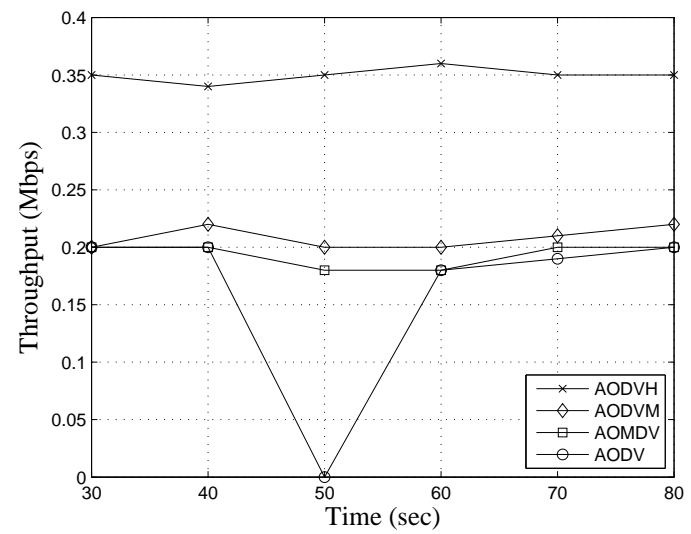

Fig. 8. Throughput vs. Time ("FSO Only" path for AODVH).

Fig. 9 shows the throughput of AODVH (hybrid paths) with other protocols. We observe that the alternate RF paths are picked when FSO links becomes unavailable in AODVH, resulting in AODVH performance being similar to AODVM and AOMDV.

From the above results, we observe that AODVH achieves better performance than other three protocols (AODV, AOMDV and AODVM) in varying mobility scenarios in terms of packet loss, end-to-end delay, route discovery frequency, packet delivery ratio, overhead and throughput.

\section{CONCLUSION}

In this paper, we proposed and evaluated a novel multipath on-demand Ad Hoc routing protocol for Disaster Area Wireless Network, consisting of hybrid nodes using FSO and RF links. The proposed protocol, called AODVH, prefers high bandwidth routes consisting of "FSO only" links over lower bandwidth hybrid routes with FSO and RF links.

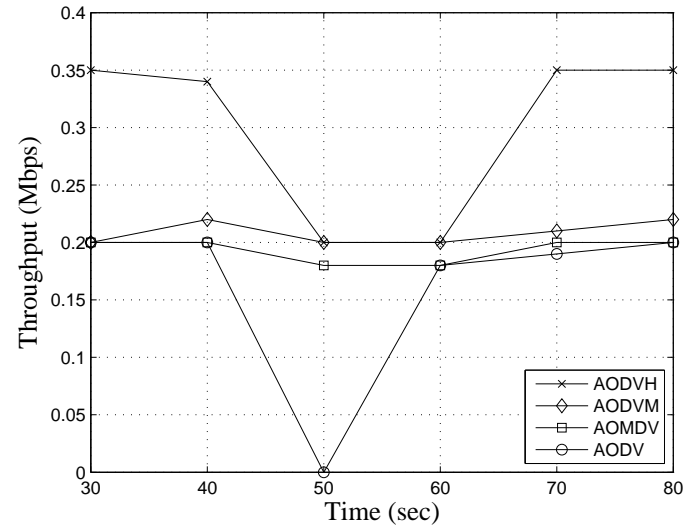

Fig. 9. Throughput vs. Time (hybrid path for AODVH).

Results validated that the multipath feature of AODVH significantly minimized packet loss, end-to-end delay, routing overhead and maximized throughput when compared to AODV, AOMDV and AODVM. In addition to disaster recovery, DAWN can be very useful in military and exploration missions, home area wireless networking, networking intelligent devices, sensors, mobile robots, and on-the-fly conference applications.

\section{REFERENCES}

[1] P. Yan, J. J. S. Jr., H. H. Refai, and P. G. LoPresti, "Enhancing mobile ad hoc networks with free-space optics," Optical Engineering, vol. 46, no. 8, pp. 1-7, August 2007.

[2] S. J. Lee and M. Gerla, "Split multipath routing with maximally disjoint paths in ad hoc networks," in IEEE International Conference on Communications, June 11 - 14, 2001, pp. 3201-3205.

[3] S. Gurumani, H. Moradi, H. H. Refai, M. Atiquzzaman, and P. G. LoPresti, "Dynamic path reconfiguration among hybrid FSO/RF nodes," in GLOBECOM, New Orleans, LO, 30 Nov - 4 Dec 2008.

[4] S. Adibi and S. Erfani, "A multipath routing survey for mobile ad hoc networks," in IEEE CCNC, Las Vegas, USA, 8-10 January 2006, pp. 984-988.

[5] D. Johnson, Y. Hu, and D. Maltz, "The Dynamic Source Routing Protocol (DSR) for Mobile Ad Hoc Networks for IPv4," RFC 4728, February 2007.

[6] T. Clausen and P. Jacquet, "Optimized Link State Routing Protocol," RFC 3626, October 2003.

[7] C. Perkins, E. Belding-Royer, and S. Das, "Ad hoc On-Demand Distance Vector routing," RFC 3561, July 2003.

[8] M. K. Marina and S. R. Das, "Ad hoc on-demand multipath distance vector routing," Wireless Communication and Mobile Computing, vol. 1, no. 6, pp. 969-988, 2006.

[9] Z. Ye, S. V. Krishnamurthy, and S. K. Tripathi, "A framework for reliable routing in mobile ad hoc networks," in IEEE INFOCOM, San Francisco, CA, 30 March - 3 April 2003, pp. 270-280.

[10] M. Bilgi and M. Yuksel, "Multi-element free-space-optical spherical structures with intermittent connectivity patterns," in INFOCOM, Phoenix, AZ, 13-18 April 2008, pp. 233-236.

[11] M. Yuksel, J. Akella, S. Kalyanaraman, and P. Dutta, "Free spaceoptical mobile ad-hoc networks: Auto-configurable building blocks," ACM/Springer Wirless Networks, vol. 15, no. 3, pp. 295-312, April 2009.

[12] C. E. Perkins and P. Bhagwat, "Highly dynamic destination-sequenced distance-vector routing (dsdv) for mobile computers," in ACM Sigcomm, London, UK, 31 August - 2 September 1994.

[13] K. Fall and K. Varadhan, "The ns manual," Ns Manual, 2002.

[14] J. Broch, D. Moltz, D. Johnson, Y.-C. Hu, and J. Jetcheva, "A performance comparison of multi-hop wireless ad hoc network routing protocols," in IEEE/ACM MobiCom, Dallas, Texas, 25-30 October 1998, pp. 85-97. 